\title{
From geometry to invertibility preservers
}

\author{
by \\ Hans Havlicek (Wien) and Peter Šemrl (Ljubljana)
}

\begin{abstract}
We characterize bijections on matrix spaces (operator algebras) preserving full rank (invertibility) of differences of matrix (operator) pairs in both directions.
\end{abstract}

1. Introduction. Marcus and Purves [19] proved that every unital invertibility preserving linear map on a matrix algebra is either an inner automorphism or an inner anti-automorphism. One of the equivalent formulations of the Gleason-Kahane-Żelazko theorem [6, 16, 25] states that every unital linear functional on a complex unital Banach algebra $\mathcal{A}$ sending every invertible element to a nonzero scalar is multiplicative. Equivalently, if a linear functional $f: \mathcal{A} \rightarrow \mathbb{C}$ maps every element $a \in \mathcal{A}$ into its spectrum $\sigma(a)$, then $f$ is multiplicative. These two results motivated Kaplansky to formulate the question under which conditions an invertibility preserving linear unital map between two algebras must be a Jordan homomorphism [17]. A lot of work has been done on this problem (see the surveys [1, 3, 22]). We will mention here only the results that are relevant to our paper.

Let $X$ be a complex Banach space and $B(X)$ the algebra of all bounded linear operators on $X$. In 1986 Jafarian and Sourour [15] proved that every surjective unital linear map $\phi: B(X) \rightarrow B(X)$ preserving invertibility in both directions, i.e., having the property that $A$ is invertible if and only if $\phi(A)$ is invertible, is either of the form $\phi(A)=T A T^{-1}, A \in B(X)$, for some invertible $T \in B(X)$, or of the form $\phi(A)=T A^{\prime} T^{-1}, A \in B(X)$, for some invertible bounded linear operator $T: X^{\prime} \rightarrow X$. Here, $A^{\prime}$ denotes the adjoint of $A$ and $X^{\prime}$ the dual of $X$. Under the additional assumption of injectivity the assumption of preserving invertibility in both directions can be relaxed to preserving invertibility in one direction only [23]. The proof of the result of Jafarian and Sourour was simplified in [21]. It is rather easy to see that

2000 Mathematics Subject Classification: 47B49, 15A04.

Key words and phrases: adjacency, full rank, invertibility, preserver.

The first author acknowledges the hospitality of the Department of Mathematics at the University of Ljubljana where most of the work was carried out. The second author was partially supported by a grant from the Ministry of Science of Slovenia. 
a linear map $\phi: B(X) \rightarrow B(X)$ is unital and preserves invertibility in both directions if and only if $\phi$ preserves the spectrum, that is, $\sigma(\phi(A))=\sigma(A)$ for every $A \in B(X)$.

An interesting extension of the Gleason-Kahane-Żelazko theorem was obtained by Kowalski and Słodkowski [18]. They proved that every functional $f$ on a complex Banach algebra $\mathcal{A}$ (they did not assume the linearity of $f$ ) satisfying $f(a)-f(b) \in \sigma(a-b), a, b \in \mathcal{A}$, is linear and multiplicative up to the constant $f(0)$. Thus, they replaced the two conditions in the GleasonKahane-Żelazko theorem, linearity and the condition $f(a) \in \sigma(a), a \in \mathcal{A}$, by a single weaker assumption and got essentially the same conclusion.

In view of this result it is natural to ask if we can do the same with the above mentioned results on invertibility preserving maps on matrix and operator algebras. Can we replace linearity and invertibility preserving by a single weaker condition similar to the one in the Kowalski-Słodkowski theorem? More precisely, can we characterize bijective maps on matrix algebras and operator algebras satisfying the condition that $\phi(a)-\phi(b)$ is invertible if and only if $a-b$ is?

The result of Kowalski and Słodkowski depends heavily on deep results from analysis. We will answer the above question using results from geometry. We should first mention that there is an essential difference between the finite and infinite-dimensional cases. In the finite-dimensional case our condition will imply, up to a translation, the semilinearity of the maps under consideration, while in the infinite-dimensional case the elementary automatic continuity methods will imply the linearity or conjugate-linearity up to a translation. Moreover, in the finite-dimensional case it makes sense to extend our result from matrix algebras of square matrices to spaces of rectangular matrices. Then, of course, the condition of invertibility will be replaced by the condition of being of full rank.

Our strategy when considering bijective maps $\phi$ on matrix spaces (operator algebras) satisfying the condition that $\phi(A)-\phi(B)$ is of full rank (invertible) if and only if $A-B$ is of full rank (invertible) will be to prove first that such maps preserve adjacency in both directions. Recall that two matrices or operators $A$ and $B$ are adjacent if $A-B$ is of rank one. Then we will apply the so called fundamental theorem of geometry of matrices (or its analogue for operators) to complete the proof. This connects our results with the geometry of Grassmann spaces. Let us briefly describe this connection.

Let $M_{m, n}, m, n \geq 2$, be the linear space of all $m \times n$ matrices over a field $\mathbb{F}$. If $\sigma$ is an automorphism of the field $\mathbb{F}$ and $A=\left[a_{i j}\right] \in M_{m, n}$ then we denote by $A_{\sigma}$ the matrix obtained from $A$ by applying $\sigma$ entrywise, $A_{\sigma}=\left[\sigma\left(a_{i j}\right)\right]$. The fundamental theorem of geometry of matrices states that every bijective map $\phi: M_{m, n} \rightarrow M_{m, n}$ preserving adjacency in both directions is of the 
form $A \mapsto T A_{\sigma} S+R$, where $T$ is an invertible $m \times m$ matrix, $S$ is an invertible $n \times n$ matrix, $R$ is an $m \times n$ matrix, and $\sigma$ is an automorphism of the underlying field. If $m=n$, then we have the additional possibility that $\phi(A)=T A_{\sigma}^{t} S+R$ where $T, S, R$ and $\sigma$ are as above, and $A^{t}$ denotes the transpose of $A$. This theorem and its analogues for hermitian matrices, symmetric matrices, and skew-symmetric matrices were proved by Hua [7][14] under some mild technical assumptions that were later proved to be superfluous (see [24]).

Let $m, n$ be integers $\geq 2$. We will consider the Grassmann space whose "points" are vector subspaces of $\mathbb{F}^{m+n}$ of dimension $m$. Chow [4] studied bijective maps on the Grassmann space preserving adjacent pairs of points in both directions. Recall that $m$-dimensional subspaces $U$ and $V$ are adjacent if $\operatorname{dim}(U+V)=m+1$. Now, to each $m$-dimensional subspace $U$ of $\mathbb{F}^{m+n}$ we can associate an $m \times(m+n)$ matrix whose rows are coordinates of vectors that form a basis of $U$. Each $m \times(m+n)$ matrix will be written in block form [ $\left.\begin{array}{ll}X & Y\end{array}\right]$, where $X$ is an $m \times n$ matrix and $Y$ is an $m \times m$ matrix. Two

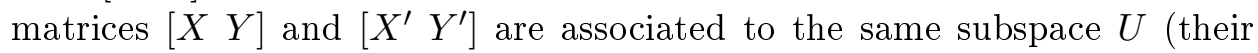
rows represent two bases of $U$ ) if and only if $\left[\begin{array}{ll}X & Y\end{array}\right]=P\left[\begin{array}{ll}X^{\prime} & \left.Y^{\prime}\right] \text { for some }\end{array}\right.$ invertible $m \times m$ matrix $P$. If this is the case, then $Y$ is invertible if and only if $Y^{\prime}$ is invertible. So, we have associated to each point in a Grassmann space a (not uniquely determined) matrix $[X Y]$. If $Y$ is singular, we say that the corresponding point in the Grassmann space is at infinity. Otherwise, we observe that this point can also be represented by the matrix $\left[\begin{array}{lll}Y^{-1} X & I\end{array}\right]$. The matrix $Y^{-1} X$ is uniquely determined by the point in the Grassmann space. So, if $U$ and $V$ are two $m$-dimensional subspaces that are finite points in the Grassmann space, then they can be represented by two uniquely determined $m \times n$ matrices $T$ and $S$, and it is easy to see that the subspaces $U$ and $V$ are adjacent if and only if the matrices $T$ and $S$ are adjacent. Using this connection it is possible to deduce the result of Chow on bijective maps on a Grassmann space preserving adjacency in both directions from the fundamental theorem of geometry of matrices (see [24]).

If we consider the special case when $m=n$ and replace, in the fundamental theorem of geometry of matrices, the condition of preserving adjacent pairs of matrices by our assumption of preserving the pairs $A, B$ with $\operatorname{rank}(A-B)=n$, then this corresponds to the study of bijective maps on the Grassmann space of all vector subspaces of $\mathbb{F}^{2 n}$ of dimension $n$ that preserve the complementarity of subspaces. Such maps were studied by Blunck and the first author [2]. We suspect that this result can be deduced from our result and the other way around, but we also believe that it is easier to prove each of them separately. Namely, to prove any of these two implications seems to be difficult because of the points at infinity. 
Now we state our main results. In the finite-dimensional case we will consider bijective maps on $m \times n$ matrices preserving pairs of matrices whose difference has a full rank. Of course, if we have such a map $\phi$ then the map $\psi: M_{n, m} \rightarrow M_{n, m}$ defined by $\psi(A)=\left(\phi\left(A^{t}\right)\right)^{t}$ has the same properties. Thus, when studying such maps there is no loss of generality in assuming that $m \geq n$. We will do this throughout the paper. A matrix $A \in M_{m, n}$ is said to be of full rank if $\operatorname{rank} A=n$. Let $A, B \in M_{m, n}$. We write $A \triangle B$ if $A-B$ is of full rank.

THEOREM 1.1. Let $\mathbb{F}$ be a field with at least three elements and $m, n$ integers with $m \geq n \geq 2$. Assume that $\phi: M_{m, n} \rightarrow M_{m, n}$ is a bijective map such that for every pair $A, B \in M_{m, n}$ we have $A \triangle B$ if and only if $\phi(A) \triangle \phi(B)$. Then there exist an invertible $m \times m$ matrix $T$, an invertible $n \times n$ matrix $S$, an $m \times n$ matrix $R$, and an automorphism $\sigma: \mathbb{F} \rightarrow \mathbb{F}$ such that

$$
\phi(A)=T A_{\sigma} S+R
$$

for every $A \in M_{m, n}$. If $m=n$, then we have the additional possibility that

$$
\phi(A)=T A_{\sigma}^{t} S+R, \quad A \in M_{n, n},
$$

where $T, S, R \in M_{n, n}$ with $T$ and $S$ invertible, and $\sigma$ is an automorphism of $\mathbb{F}$.

THEOREM 1.2. Let $H$ be an infinite-dimensional complex Hilbert space and $B(H)$ the algebra of all bounded linear operators on $H$. Assume that $\phi: B(H) \rightarrow B(H)$ is a bijective map such that for every pair $A, B \in B(H)$ the operator $A-B$ is invertible if and only if $\phi(A)-\phi(B)$ is invertible. Then there exist $R \in B(H)$ and invertible $T, S \in B(H)$ such that $\phi$ has one of the following forms:

$$
\begin{aligned}
& \phi(A)=T A S+R, \\
& \phi(A)=T A^{t} S+R, \\
& \phi(A)=T A^{*} S+R, \\
& \phi(A)=T\left(A^{t}\right)^{*} S+R,
\end{aligned}
$$

for every $A \in B(H)$. Here, $A^{t}$ and $A^{*}$ denote the transpose with respect to an arbitrary but fixed orthonormal basis, and the usual adjoint of $A$ in the Hilbert space sense, respectively.

The converses of both theorems obviously hold true. In the second section we will prove the finite-dimensional case and in the third one the infinitedimensional case. These two sections can be read independently.

2. The finite-dimensional case. In this section we will consider matrices over a field $\mathbb{F}$ with at least three elements. At a certain point in the proof of our first main theorem we will identify $m \times n$ matrices with linear operators from $\mathbb{F}^{n}$ into $\mathbb{F}^{m}$. For such operators we have the following simple lemma. 
Lemma 2.1. Let $T, S: \mathbb{F}^{n} \rightarrow \mathbb{F}^{m}$ be nonzero linear operators and assume that $T$ has at least two-dimensional image. Then we can find linearly independent vectors $x, y \in \mathbb{F}^{n}$ such that $T x$ and Sy are linearly independent.

Proof. Take any $y \in \mathbb{F}^{n}$ such that $S y \neq 0$. The set of all vectors $z \in \mathbb{F}^{n}$ with $T z$ and $S y$ linearly dependent is a proper subspace of $\mathbb{F}^{n}$, since the image of $T$ is not contained in the span of $S y$. There exist at least two linearly independent vectors of $\mathbb{F}^{n}$ which are not in this subspace. One of them is linearly independent of $y$ and gives the required vector $x$.

We have two relations on $M_{m, n}$ : adjacency and $\triangle$. The following result connecting them is the key step in our proof. We believe it is of some independent interest.

Proposition 2.2. Let $A, B \in M_{m, n}$ with $A \neq B$. Then the following are equivalent:

1. $A$ and $B$ are adjacent.

2. There exists $R \in M_{m, n}, R \neq A, B$, such that for every $X \in M_{m, n}$ the relation $X \triangle R$ yields $X \triangle A$ or $X \triangle B$.

Proof. Note that neither of the above conditions is affected if we replace $A$ and $B$ by $P A Q-C$ and $P B Q-C$, respectively, where $P$ and $Q$ are invertible matrices of the appropriate size and $C$ is any $m \times n$ matrix. Thus if the rank distance between $A$ and $B$ equals $r$ then we may assume with no loss of generality that $A=0$ and

$$
B=\left(\begin{array}{ll}
I & 0 \\
0 & 0
\end{array}\right)
$$

where $I$ is the $r \times r$ identity matrix and the zeros stand for zero matrices of appropriate size.

Assume first that $A$ and $B$ are adjacent. So, without loss of generality, we have $A=0$ and $B=E_{11}$. Set $R=\lambda E_{11}$, where $\lambda$ is a scalar different from 0 and 1 , and $E_{11}$ denotes the matrix with the $(1,1)$-entry equal to 1 and all other entries zero. Now let $X \triangle R$. That means that $X-R$ is of full rank, or equivalently, $X-R$ has at least one invertible $n \times n$ submatrix. We consider two possibilities. First assume that one of these submatrices does not contain the first row. In this case $X$ is of full rank and thus $X \triangle A$. Otherwise any such submatrix contains the first row and we choose one of them. We will prove that at least one of the corresponding $n \times n$ submatrices of $X-A=X$ and $X-B$ is invertible. So we restrict our attention to these $n \times n$ submatrices. In other words we deal only with the square case $m=n$. Hence $X-\lambda E_{11}$ is an invertible square matrix. If the first row of $E_{11}$, i.e. $(1,0, \ldots, 0)$, is in the subspace spanned by rows $2,3, \ldots, n$ of $X$ then $X-\lambda E_{11}-\mu E_{11}$ is invertible for all $\mu \in \mathbb{F}$, otherwise this holds for all but one $\mu \in \mathbb{F}$. Therefore 
$X-\lambda E_{11}-\mu E_{11}$ is invertible for $\mu=-\lambda$ or $\mu=-\lambda+1$. Equivalently, at least one of $X=X-A$ or $X-E_{11}=X-B$ is invertible, as desired. This completes the proof of the first implication.

To prove the other direction we identify $m \times n$ matrices with linear operators from $\mathbb{F}^{n}$ into $\mathbb{F}^{m}$. We assume that $A=0$ and $B: \mathbb{F}^{n} \rightarrow \mathbb{F}^{m}$ is a linear operator whose image is at least two-dimensional. Let $R: \mathbb{F}^{n} \rightarrow \mathbb{F}^{m}$ be any linear operator, $R \neq 0, B$. We have to find a linear operator $X: \mathbb{F}^{n} \rightarrow \mathbb{F}^{m}$ such that $X-R$ is injective while $X$ and $X-B$ are not.

The first possibility we will treat is that $B-R$ or $R$ has rank at least two. Then, by Lemma 2.1, we can find linearly independent $x, y \in \mathbb{F}^{n}$ such that $B x-R x$ and $R y$ are linearly independent. We first define $X$ on the linear span of $x$ and $y$. We set $X x=B x$ and $X y=0$. No matter how we extend $X$ to the whole space these two equalities guarantee that $X-B$ and $X$ will not be injective. Now, $(X-R) x=B x-R x$ and $(X-R) y=-R y$ are linearly independent. It is now obvious that we can extend $X$ to the whole $\mathbb{F}^{n}$ so that the resulting $X-R$ is injective.

It remains to consider the case when both $B-R$ and $R$ are of rank one. By our assumption, $B$ is of rank two. Hence $B=R+(B-R)$ implies that the ranges of $B-R$ and $R$ meet in 0 only. So, if we choose any $x, y \in \mathbb{F}^{n}$ such that $(B-R) x \neq 0$ and $R y \neq 0$ then $(B-R) x$ and $R y$ will be linearly independent. Since $\mathbb{F}$ has at least three elements, we can choose these $x$ and $y$ to be linearly independent. Now we can proceed as above.

It is now easy to prove Theorem 1.1. Namely, if $\phi: M_{m, n} \rightarrow M_{m, n}$ is a bijective map preserving $\triangle$ in both directions, then by Proposition 2.2 it preserves adjacency in both directions. Thus, the result follows directly from the fundamental theorem of geometry of matrices.

Observe that in [2] there is no need to assume that $\mathbb{F}$ has at least three elements, due to the presence of points at infinity.

3. The infinite-dimensional case. Let $H$ be an infinite-dimensional complex Hilbert space and $x, y \in H$. The inner product of $x$ and $y$ will be denoted by $y^{*} x$. If $x$ and $y$ are nonzero vectors then $x y^{*}$ stands for the rank one bounded linear operator defined by $\left(x y^{*}\right) z=\left(y^{*} z\right) x, z \in H$. Note that every bounded rank one operator can be written in this form. Two operators $A, B \in B(H)$ are said to be adjacent if $A-B$ is an operator of rank one. We write $A \triangle B$ if $A-B$ is invertible. We start with an analogue of Proposition 2.2.

Proposition 3.1. Let $A, B \in B(H)$ with $A \neq B$. Then the following statements are equivalent:

1. A and $B$ are adjacent. 
2. There exists $R \in B(H), R \neq A, B$, such that for every $X \in B(H)$ the relation $X \triangle R$ yields $X \triangle A$ or $X \triangle B$.

Proof. Note that neither of the above conditions is affected if we replace $A$ and $B$ by $A-C$ and $B-C$, respectively, where $C$ is any bounded linear operator on $H$. Thus we may assume with no loss of generality that $A=0$.

Assume first that $A=0$ and $B$ are adjacent, that is, $B$ is of rank one. Set $R=2 B$. Suppose that $X-2 B$ is invertible. Then

$$
X-2 B-\lambda B=(X-2 B)\left(I-\lambda(X-2 B)^{-1} B\right)
$$

is invertible if and only if $I-\lambda S$ is invertible, where $S=(X-2 B)^{-1} B$ is an operator of rank one. Every operator of rank one has at most one nonzero complex number in its spectrum. Hence, $X-2 B-(-2 B)=X$ is invertible or $X-2 B-(-B)=X-B$ is invertible. This completes the proof of one direction.

Assume now that $A=0$ and $B$ is an operator whose image is at least two-dimensional. We have to prove that for every $R \in B(H), R \neq 0, B$, there exists $X \in B(H)$ such that $X-R$ is invertible and $X$ is singular and $X-B$ is singular. So, let $R \in B(H) \backslash\{0, B\}$.

In the next step we will prove that there exist $x, z \in H$ such that $x$ and $z$ are linearly independent and $B z-R z$ and $R x$ are linearly independent. It is enough to show that we can find $x, z \in H$ such that $B z-R z$ and $R x$ are linearly independent. For if $x$ and $z$ are linearly dependent, we can choose $u \in H$ linearly independent of $x$. Then $z+\lambda u$ and $x$ are linearly independent for all nonzero $\lambda$, and for all $\lambda$ 's small enough the vectors $B(z+\lambda u)-$ $R(z+\lambda u)=B z-R z+\lambda(B u-R u)$ and $R x$ are linearly independent as well.

So, let us show that such $x$ and $z$ exist. Assume on the contrary that $B z-R z$ and $R x$ are linearly dependent for every $x$ and $z$. Then $B-R$ and $R$ are rank one operators with the same one-dimensional image. It follows that $B=0$ or $B$ is of rank one, a contradiction.

Now, we define $W$ to be the orthogonal complement of the linear span of $x$ and $z$, where $x$ and $z$ are as in the previous paragraph, and $Z$ to be the orthogonal complement of $R x$ and $B z-R z$. Then there exists a bounded invertible linear operator $U: W \rightarrow Z$. Define $X \in B(H)$ by

$$
X x=0, \quad X z=B z, \quad X u=U u+R u, \quad u \in W .
$$

Because of the first two equalities the operators $X$ and $X-B$ are singular. Since $(X-R) x=-R x,(X-R) z=B z-R z$, and $(X-R) u=U u, u \in W$, the operator $X-R$ is invertible, as desired.

We continue with some technical lemmas.

Lemma 3.2. Let $B, C \in B(H)$. Assume that for every invertible $A \in$ $B(H)$ the operator $A-B$ is invertible if and only if $A-C$ is invertible. Then $B=C$. 
Proof. Let $\lambda$ be any complex number satisfying $|\lambda|>\|B\|,\|C\|$, and $x, y \in H$ any vectors such that $y^{*} x=0$. Then $\lambda\left(I+x y^{*}\right)$ is invertible because $\left(I+x y^{*}\right)\left(I-x y^{*}\right)=I$. Hence, $\lambda I+\lambda x y^{*}-B$ is invertible if and only if $\lambda I+\lambda x y^{*}-C$ is. On the other hand,

$$
\begin{aligned}
\lambda I+\lambda x y^{*}-B & =\left(I+x y^{*}\right)\left(\lambda I-B+x y^{*} B\right) \\
& =\left(I+x y^{*}\right)\left(I+x y^{*} B(\lambda I-B)^{-1}\right)(\lambda I-B)
\end{aligned}
$$

is invertible if and only if $I+x y^{*} B(\lambda I-B)^{-1}$ is. Thus, $I+x y^{*} B(\lambda I-B)^{-1}$ is invertible if and only if $I+x y^{*} C(\lambda I-C)^{-1}$ is, or equivalently, for every scalar $\lambda$ with $|\lambda|>\|B\|,\|C\|$, and every pair of vectors $x, y \in H$ with $y^{*} x=0$ we have

$$
y^{*} B(\lambda I-B)^{-1} x=-1 \Leftrightarrow y^{*} C(\lambda I-C)^{-1} x=-1 .
$$

Fix $\lambda$. Then $y^{*} T x=0$ for every pair of orthogonal vectors $x$ and $y$, where $T=B(\lambda I-B)^{-1}-C(\lambda I-C)^{-1}$. It follows that $T=\mu I$ for some scalar $\mu$. Thus, for every $\lambda$ with $|\lambda|>\|B\|,\|C\|$ we have

$$
B(\lambda I-B)^{-1}-C(\lambda I-C)^{-1}=g(\lambda) I
$$

for some $g(\lambda) \in \mathbb{C}$. Obviously, $g(\lambda)$ is holomorphic outside the disk centered at 0 with radius $\max \{\|B\|,\|C\|\}$. Expanding the above analytic functions in series and comparing the coefficients we get

$$
B=C+\mu I
$$

for some complex number $\mu$. Our assumption implies that $\sigma(B) \backslash\{0\}=$ $\sigma(C) \backslash\{0\}$. Here $\sigma(B)$ denotes the spectrum of $B$. It follows that $\mu=0$, as desired.

Lemma 3.3. Let $A, B \in B(H)$ be invertible operators. Assume that for every rank one operator $x y^{*} \in B(H)$ the operator $A-x y^{*}$ is invertible if and only if $B-x y^{*}$ is invertible. Then $A=B$.

Proof. Our assumptions imply that for every pair of vectors $x, y$ the operator $I-x y^{*} A^{-1}$ is invertible if and only if $I-x y^{*} B^{-1}$ is, or equivalently, $y^{*} A^{-1} x=1$ if and only if $y^{*} B^{-1} x=1$. By linearity we have $y^{*} A^{-1} x=y^{*} B^{-1} x$ for any $x, y \in H$, and therefore, $A^{-1}=B^{-1}$. It follows that $A=B$.

Recall that an additive map $T: H \rightarrow H$ is called semilinear if there is an automorphism $\sigma: \mathbb{C} \rightarrow \mathbb{C}$ such that $T(\lambda x)=\sigma(\lambda) T x$ for every $\lambda \in \mathbb{C}$ and every $x \in H$. Now we are ready to prove Theorem 1.2.

Let $\phi: B(H) \rightarrow B(H)$ be a bijective map such that for every pair $A, B \in B(H)$ the operator $A-B$ is invertible if and only if $\phi(A)-\phi(B)$ is. 
After replacing $\phi$ by $A \mapsto \phi(A)-\phi(0)$ we may assume that $\phi(0)=0$. Then $\phi(I)$ is invertible. Replacing $\phi$ by $A \mapsto \phi(I)^{-1} \phi(A)$ we may further assume that $\phi(I)=I$.

According to Proposition 3.1, $\phi$ preserves adjacency in both directions. Every rank one operator is adjacent to zero, every rank two operator is adjacent to some rank one operator, etc. Consequently, $\phi$ maps the subspace $F(H) \subset B(H)$ of all finite rank operators onto itself. So, we can apply Theorem 1.5 from [20] to conclude that there exist bijective semilinear maps $T, S: H \rightarrow H$ (with the same associated automorphism) such that either $\phi\left(x y^{*}\right)=(T x)(S y)^{*}, x, y \in H$, or $\phi\left(x y^{*}\right)=(S y)(T x)^{*}, x, y \in$ $H$. The second case can be reduced to the first one if we replace $\phi$ by $A \mapsto \phi(A)^{*}, A \in B(H)$. So, we may assume that the first possibility holds true.

Using $\phi(I)=I$ and our assumptions we conclude that $I-x y^{*}$ is invertible if and only if $I-(T x)(S y)^{*}$ is, for any $x, y \in H$. Thus, $y^{*} x=1$ if and only if $(S y)^{*}(T x)=1$, and by semilinearity,

$$
(S y)^{*}(T x)=0 \Leftrightarrow y^{*} x=0, \quad x, y \in H .
$$

Thus, the semilinear maps $T$ and $S$ and their inverses carry closed hyperplanes (every closed hyperplane is the orthogonal complement of some nonzero vector) onto closed hyperplanes. Hence, by [5, Lemmas 2 and 3], $S$ and $T$ are both linear bounded or both conjugate-linear bounded. Thus, we have $\phi\left(x y^{*}\right)=T\left(x y^{*}\right) R$, where $T$ and $R=S^{*}$ are bounded, invertible, and either both linear, or both conjugate-linear. Assume they are both conjugate-linear. Choosing an orthonormal basis we define $K: H \rightarrow H$ to be the conjugate-linear bijection which maps each vector $x$ into a vector whose coordinates are obtained from the coordinates of $x$ by complex conjugation. Of course, $K^{2}=I$, the product of two conjugate-linear maps is linear, and $K\left(x y^{*}\right) K=\left(\left(x y^{*}\right)^{*}\right)^{t}$, where the transpose is taken with the respect to the chosen basis. Replacing $\phi$ by $A \mapsto\left(\phi(A)^{t}\right)^{*}, A \in B(H)$, we reduce the conjugate-linear case to the linear one.

So, we may assume that $\phi\left(x y^{*}\right)=T\left(x y^{*}\right) R$, where $T$ and $R=S^{*}$ are bounded invertible linear operators. From $(S y)^{*}(T x)=1 \Leftrightarrow y^{*} x=1$ and linearity we get $(S y)^{*}(T x)=y^{*} x, x, y \in H$, which further implies that $T$ is the inverse of $R$. Composing $\phi$ with a similarity transformation we may further assume that $\phi\left(x y^{*}\right)=x y^{*}, x, y \in H$.

Let $A \in B(H)$ be invertible. Applying Lemma 3.3 with $B=\phi(A)$ we see that $\phi(A)=A$.

Finally, let $B \in B(H)$ be any operator and set $C=\phi(B)$. Using Lemma 3.2 we conclude that $\phi(B)=B$. This completes the proof. 


\section{References}

[1] B. Aupetit, Propriétés spectrales des algèbres de Banach, Lecture Notes in Math. 735, Springer, New York, 1979.

[2] A. Blunck and H. Havlicek, On bijections that preserve complementarity of subspaces, Discrete Math. 301 (2005), 46-54.

[3] M. Brešar and P. Šemrl, Spectral characterization of idempotents and invertibility preserving maps, Expo. Math. 17 (1999), 185-192.

[4] W.-L. Chow, On the geometry of algebraic homogeneous spaces, Ann. of Math. 50 (1949), 32-67.

[5] P. A. Fillmore and W. E. Longstaff, On isomorphisms of lattices of closed subspaces, Canad. J. Math. 36 (1984), 820-829.

[6] A. M. Gleason, A characterization of maximal ideals, J. Anal. Math. 19 (1967), 171-172.

[7] L. K. Hua, Geometries of matrices I. Generalizations of von Staudt's theorem, Trans. Amer. Math. Soc. 57 (1945), 441-481.

[8] -, Geometries of matrices $I_{1}$. Arithmetical construction, ibid. 57 (1945), 482-490.

[9] -, Geometries of symmetric matrices over the real field I, Dokl. Akad. Nauk SSSR 53 (1946), 95-97.

[10] -, Geometries of symmetric matrices over the real field II, ibid. 53 (1946), 195-196.

[11] - Geometries of matrices II. Study of involutions in the geometry of symmetric matrices, Trans. Amer. Math. Soc. 61 (1947), 193-228.

[12] - Geometries of matrices III. Fundamental theorems in the geometries of symmetric matrices, ibid. 229-255.

[13] -, Geometry of symmetric matrices over any field with characteristic other than two, Ann. of Math. 50 (1949), 8-31.

[14] - A theorem on matrices over a sfield and its applications, Acta Math. Sinica 1 (1951), 109-163.

[15] A. Jafarian and A. R. Sourour, Spectrum preserving linear maps, J. Funct. Anal. 66 (1986), 255-261.

[16] J.-P. Kahane and W. Żelazko, A characterization of maximal ideals in commutative Banach algebras, Studia Math. 29 (1968), 339-343.

[17] I. Kaplansky, Algebraic and Analytic Aspects of Operator Algebras, Amer. Math. Soc., Providence, 1970.

[18] S. Kowalski and Z. Słodkowski, A characterization of multiplicative linear functionals in Banach algebras, Studia Math. 68 (1980), 215-223.

[19] M. Marcus and R. Purves, Linear transformations on algebras of matrices: The invariance of the elementary symmetric functions, Canad. J. Math. 11 (1959), 383396.

[20] T. Petek and P. Šemrl, Adjacency preserving maps on matrices and operators, Proc. Roy. Soc. Edinburgh Sect. A 132 (2002), 661-684.

[21] P. Šemrl, Invertibility preserving linear maps and algebraic reflexivity of elementary operators of length one, Proc. Amer. Math. Soc. 130 (2002), 769-772.

[22] A. R. Sourour, The Gleason-Kahane-Żelazko theorem and its generalizations, in: Banach Center Publ. 30, Inst. Math., Polish Acad. Sci., 1994, 327-331.

[23] -, Invertibility preserving linear maps on $\mathcal{L}(X)$, Trans. Amer. Math. Soc. 348 (1996), 13-30.

[24] Z.-X. Wan, Geometry of Matrices, World Sci., Singapore, 1996. 
[25] W. Żelazko, A characterization of multiplicative linear functionals in complex Banach algebras, Studia Math. 30 (1968), 83-85.

Institut für Diskrete Mathematik und Geometrie Technische Universität Wien

Wiedner Hauptstrasse 8-10

A-1040 Wien, Austria

E-mail: havlicek@geometrie.tuwien.ac.at
Department of Mathematics University of Ljubljana Jadranska 19

SI-1000 Ljubljana, Slovenia E-mail: peter.semrl@fmf.uni-lj.si

Received June 16, 2005

Revised version January 17, 2006 